\title{
Gene Therapy Arrives
}

apply to employers with fewer than 15 employees and does not cover insurance for long-term care, life or disability. It also does not apply to people employed and insured by the military's Tricare system, such as Rylan Gorby. When his son's genome was sequenced, researchers also obtained permission to sequence Rylan's genome, to determine if he was a carrier for the rare hemoglobin condition. Because it manifests itself only in childhood, Gorby decided taking the test was worth the risk of possible discrimination.

Cost is another consideration. Clinical sequencing is still about $\$ 500$ to $\$ 800$, and interpretation can be upward of $\$ 1,000$, according to Brigham and Women's Green. For families who can't afford health insurance, this is out of reach. Some experts have also raised concerns that genetic testing could lead to a lot of follow-up testing with specialists, which could overburden an already resource-strapped health care system. If sequencing turns out to save money in the long run, insurance companies may cover it, but there's no guarantee.

Yet another problem is that the majority of the sequencing to date has been done in babies whose families are well-off and white, raising concerns that this could become the province of only the privileged. And the racial homogeneity could skew the results: diseases more prevalent in Caucasian individuals could be overrepresented in test panels, whereas illnesses more common in racial minorities may be underrepresented. (New medical data projects intend to address this disparity [see "All of Us," on page S14].)

THE U.C.S.F. NSIGHT PROJECT included a working group that investigated some of these ethical and policy issues, which culminated in a 2018 report by the Hastings Center, a bioethics nonprofit in Garrison, N.Y. The report concluded that newborn sequencing has many benefits in helping diagnose sick babies and could expand the number of conditions that meet the stringent newborn screening criteria. But using genome sequencing as a replacement for newborn screening is "at best premature," the authors say, and direct-to-consumer sequencing should not be used for diagnosis or screening purposes.

Barbara Koenig, a professor of medical anthropology and bioethics at U.C.S.F. and one of the report's co-authors, underscores the fact that sequencing, while promising, is not yet mature enough to be routinely used to screen healthy children. "This is not a technology that's ready for prime time for use in healthy infants," Koenig says.

Despite these concerns, the era of newborn sequencing is now upon us, and the practice will likely become more widespread as costs come down and the results become more accurate and useful. In the meantime, the risks and benefits of sequencing must be weighed on an individual basis. Extremely sick newborns are a completely different case from apparently healthy children of worried parents susceptible to marketing from genetic-testing firms.

For Mitchell Gorby, sequencing was certainly worth it. Two months after leaving the hospital, he is doing fine and has doubled his weight. His parents are settling into their new routine, somewhat sleep-deprived, but happy to be home with their healthy baby boy.

Tanya Lewis is an associate editor who covers health and medicine at Scientific American.

\section{After false starts, drugs that manipulate the code of life are finally changing lives}

\section{By Jim Daley}

The idea for gene therapy - a type of DNA-based medicine that inserts a healthy gene into cells to replace a mutated, diseasecausing variant-was first published in 1972. After decades of disputed results, treatment failures and some deaths in experimental trials, the first gene therapy drug, for a type of skin cancer, was approved in China in 2003. The rest of the world was not easily convinced of the benefits, however, and it was not until 2017 that the U.S. approved one of these medicines. Since then, the pace of approvals has accelerated quickly. At least nine gene therapies have been approved for certain kinds of cancer, some viral infections and a few inherited disorders. A related drug type interferes with faulty genes by using stretches of DNA or RNA to hinder their workings. After nearly half a century, the concept of genetic medicine has become a reality.

\section{GENE INSERTION}

These treatments use a harmless virus to carry a good gene into cells, where the virus inserts it into the existing genome, canceling the effects of harmful mutations in another gene.

\section{GENDICINE: China's}

regulatory agency approved

the world's first commercially available gene therapy in 2003 to treat head and neck squamous cell carcinoma, a form of skin cancer. Gendicine is a virus engineered to carry a gene that has instructions for making a tumor-fighting protein. The virus introduces the gene into tumor cells, causing them to increase the expression of tumor-suppressing genes and immune response factors. The drug is still awaiting FDA approval. GLYBERA: The first gene therapy to be approved in the European Union treated lipoprotein lipase deficiency (LPLD), a rare inherited disorder that can cause severe pancreatitis. The drug inserted the gene for lipoprotein lipase into muscle cells. But because LPLD occurs in so few patients, the drug was unprofitable. By 2017 its manufacturer declined to renew its marketing authorization; Glybera is no longer on the market. IMLYGIC: The drug was approved in China, the U.S. and the E.U. to treat melanoma in patients who have recurring skin lesions following initial surgery. Imlygic is a modified genetic therapy inserted directly into tumors with a viral vector, where the gene replicates and produces a protein that stimulates an immune response to kill cancer cells. KYMRIAH: Developed for patients with B cell lymphoblastic leukemia, a type of cancer that affects white blood cells in children and young adults, Kymriah was approved by the FDA in 2017 and the E.U. in 2018. It works by introducing a new gene into a patient's own T cells that enables them to find and kill cancer cells. 
LUXTURNA: The drug was approved by the FDA in 2017 and in the E.U. in 2018 to treat patients with a rare form of inherited blindness called biallelic RPE65 mutation-associated retinal dystrophy. The disease affects between 1,000 and 2,000 patients in the U.S. who have a mutation in both copies of a particular gene, RPE65. Luxturna delivers a normal copy of RPE65 to patients' retinal cells, allowing them to make a protein necessary for converting light to electrical signals and restoring their vision.

\section{STRIMVELIS: About 15} patients are diagnosed in Europe every year with severe immunodeficiency from a rare inherited condition called adenosine deaminase deficiency (ADA-SCID). These patients' bodies cannot make the ADA enzyme, which is vital for healthy white blood cells. Strimvelis, approved in the E.U. in 2016 , works by introducing the gene responsible for producing ADA into stem cells taken from the patient's own marrow. The cells are then reintroduced into the patient's bloodstream, where they are transported to the bone marrow and begin producing normal white blood cells that can produce ADA.

YESCARTA: Developed to treat a cancer called large B cell lymphoma, Yescarta was approved by the FDA in 2017 and in the E.U. in 2018. It is in clinical trials in China. Large B cell lymphoma affects white blood cells called lymphocytes. The treatment, part of an approach known as
CAR-T cell therapy, uses a virus to insert a gene that codes for proteins called chimeric antigen receptors (CARs) into a patient's $T$ cells. When these cells are reintroduced into the patient's body, the CARs allow them to attach to and kill cancer cells in the bloodstream.

ZOLGENSMA: In May 2019 the FDA approved Zolgensma for children younger than two years with spinal muscular atrophy, a neuromuscular disorder that affects about one in 10,000 people worldwide. It is one of the leading genetic causes of infant mortality. Zolgensma delivers a healthy copy of the human SMN gene to a patient's motor neurons in a single treatment. ZYNTEGLO: Granted approval in the E.U. in May 2019, Zynteglo treats a blood disorder called beta thalassemia that reduces a patient's ability to produce hemoglobin, the protein in red blood cells that contains iron, leading to lifethreatening anemia. The therapy has been approved for individuals 12 years and older who require regular blood transfusions. It employs a virus to introduce healthy copies of the gene for making hemoglobin into stem cells taken from the patient.The cells are then reintroduced into the bloodstream and transported to the bone marrow, where they begin producing healthy red blood cells that can manufacture hemoglobin.

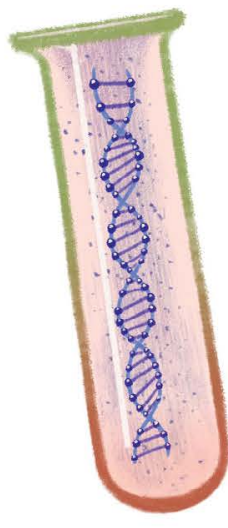

\section{GENE INTERFERENCE}

This approach uses a synthetic strand of RNA or DNA (called an oligonucleotide) that, when introduced into a patient's cell, can attach to a specific gene or its messenger molecules, effectively inactivating them. Some treatments use an antisense method, named for one DNA strand, and others rely on small interfering RNA strands, which stop instruction molecules that go from the gene to the cell's protein factories.

DEFITELIO: This drug contains a mixture of single-strand oligonucleotides obtained from the intestinal mucosa of pigs. It was approved (with limitations) in the U.S. and the E.U. in 2017 to treat severe cases of veno-occlusive disease, a disorder in which the small veins of the liver become obstructed, in patients who have received a bone marrow transplant.

EXONDYS 51: In 2016

the FDA granted approval to Exondys 51 amid some controversy regarding its efficacy; two members of the FDA review panel resigned in protest of the decision. The therapy is designed to treat a form of Duchenne muscular dystrophy caused by mutations in the RNA that codes for the protein that helps to connect muscle fibers' cytoskeletons to a surrounding matrix.

Exondys 51 is effective in treating about 13 percent of the Duchenne population. KYNAMRO: Approved by the FDA in in 2013, Kynamro is designed to inhibit-or effectively shut down production ofa protein that helps to produce low-density lipoprotein (LDL). Injected subcutaneously, this therapy is used to lower LDL levels in patients who have dangerously high cholesterol.

MACUGEN: Age-related macular degeneration is the leading cause of vision loss in people age 60 and older. It is caused by deterioration of the center of the retina due to leaking blood vessels. Approved in the U.S., Macugen inhibits these blood vessels from growing under the retina, thus treating the disorder. SPINRAZA: With its FDA approval in 2016, Spinraza became the first gene-based therapy for spinal muscular atrophy. The inherited disorder is caused by low levels of SMN, a key protein for the maintenance of motor neurons. Spinraza binds to RNA from a "backup" gene called SMN2, converting that RNA into instructions for making fully functioning SMN proteins.

Jim Daley is a freelance journalist based in Chicago. 